\title{
Well-posedness and asymptotic stability to a laminated beam in thermoelasticity of type III
}

\author{
Yue Luan, Wenjun Liu* and Gang Li \\ College of Mathematics and Statistics \\ Nanjing University of Information Science and Technology \\ Nanjing 210044, China
}

\begin{abstract}
In this paper, we study the well-posedness and asymptotic behaviour of solutions to a laminated beam in thermoelasticity of type III. We first give the well-posedness of the system by using the semigroup method. Then, we show that the system is exponentially stable under the assumption of equal wave speeds. Furthermore, it is proved that the system is lack of exponential stability for case of nonequal wave speeds. In this regard, a polynomial stability result is proved.
\end{abstract}

2000 Mathematics Subject Classification: 35B40, 35L56, 74F05, 93D20.

Keywords: laminated beam, thermoelasticity of type III, exponential decay, polynomial decay.

\section{Introduction}

In this paper, we consider a coupled system of a laminated beam with thermoelasticity of type III, which has the form

$$
\begin{cases}\rho_{1} \varphi_{t t}+G\left(\psi-\varphi_{x}\right)_{x}=0, & (x, t) \in(0,1) \times(0,+\infty), \\ I_{\rho_{1}}(3 \omega-\psi)_{t t}-D(3 \omega-\psi)_{x x}-G\left(\psi-\varphi_{x}\right)+\alpha \theta_{x}=0, & (x, t) \in(0,1) \times(0,+\infty), \\ I_{\rho_{1}} \omega_{t t}-D \omega_{x x}+G\left(\psi-\varphi_{x}\right)+\frac{4}{3} \beta_{1} \omega+\frac{4}{3} \beta_{2} \omega_{t}=0, & (x, t) \in(0,1) \times(0,+\infty), \\ \rho_{2} \theta_{t t}-\delta \theta_{x x}+\gamma(3 \omega-\psi)_{t t x}-k \theta_{t x x}=0, & (x, t) \in(0,1) \times(0,+\infty), \\ \varphi(x, 0)=\varphi_{0}(x), \psi(x, 0)=\psi_{0}(x), & x \in[0,1], \\ \omega(x, 0)=\omega_{0}(x), \theta(x, 0)=\theta_{0}(x), & x \in[0,1], \\ \varphi_{t}(x, 0)=\varphi_{1}(x), \psi_{t}(x, 0)=\psi_{1}(x), & x \in[0,1], \\ \omega_{t}(x, 0)=\omega_{1}(x), \theta_{t}(x, 0)=\theta_{1}(x), & x \in[0,1], \\ \varphi_{x}(0, t)=\varphi_{x}(1, t)=\psi(0, t)=\psi(1, t)=0, & t \in[0,+\infty), \\ \omega(0, t)=\omega(1, t)=\theta_{x}(0, t)=\theta_{x}(1, t)=0, & t \in[0,+\infty),\end{cases}
$$

where $\varphi(x, t)$ denotes the transverse displacement, $\psi(x, t)$ represents the rotation angle, $\omega(x, t)$ is proportional to the amount of slip along the interface at time $t$ and longitudinal spatial variable

${ }^{*}$ Corresponding author. $\quad$ Email address: wjliu@nuist.edu.cn (W. J. Liu). 
$x, \theta(x, t)$ is the differential temperature, respectively, and $\rho_{1}, \rho_{2}, I_{\rho_{1}}, G, D, k, \alpha, \beta_{1}, \beta_{2}, \delta, \gamma$ are positive constants. Moreover, $\sqrt{\frac{G}{\rho_{1}}}$ and $\sqrt{\frac{D}{I_{\rho_{1}}}}$ are two wave speeds.

The asymptotic behaviors of the laminated beam have been investigated by several authors over the past twenty years. Roughly speaking, laminated beam describes that two identical homogeneous beams are allowed between the beams, which were placed on top of each and a slip at the interface. These composite laminates usually have superior structural properties such as adaptability. The design of their piezoelectric materials can be invoked as both actuators and sensors. Based on the Timoshenko system, the model for this structure was first introduced by Hansen [9]. Later on, Hansen and Spies [10] studied the boundary stabilization of laminated beams with structural damping, which is

$$
\begin{cases}\rho \varphi_{t t}+G\left(\psi-\varphi_{x}\right)_{x}=0, & (x, t) \in(0,1) \times(0,+\infty), \\ I_{\rho}(3 \omega-\psi)_{t t}-D(3 \omega-\psi)_{x x}-G\left(\psi-\varphi_{x}\right)=0, & (x, t) \in(0,1) \times(0,+\infty), \\ 3 I_{\rho} \omega_{t t}-3 D \omega_{x x}+3 G\left(\psi-\varphi_{x}\right)+4 \gamma \omega+4 \beta \omega_{t}=0, & (x, t) \in(0,1) \times(0,+\infty),\end{cases}
$$

where $G, \rho, I_{\rho}, D, \gamma, \beta>0$ are the shear stiffness, the density of the beams, mass moment of inertia, flexural rigidity, adhesive stiffness of the beams and the adhesive damping parameter, respectively. In [31], the following boundary feedback controls were proposed to exponentially stabilize system (1.2):

$$
u_{1}(t)=k_{1} \varphi_{t}(1, t), u_{2}(t)=-k_{2}\left(3 \omega_{t}-\psi_{t}\right)(1, t),
$$

where $k_{1}$ and $k_{2}$ were positive constant feedback gains. Then the boundary conditions became

$$
\begin{cases}\varphi(0, t)=\psi(0, t)=\omega(0, t)=0, & t>0, \\ \psi(1, t)-\varphi_{x}(1, t)=u_{1}(t), \omega_{x}(1, t)=0,\left(3 \omega_{x}-\psi_{x}\right)(1, t)=u_{2}(t), & t \in[0,+\infty),\end{cases}
$$

and the close-loop system had both internal damping and boundary controls. They assumed that $r_{1}:=\frac{G}{\rho} \neq \frac{D}{I_{\rho}}=: r_{2}, k_{i} \neq r_{i}, i=1,2$, and found out an explicit asymptotic formula for the matrix fundamental solutions. Then they carried out the asymptotic analysis for the eigenpairs by using an invertible matrix function with an eigenvalue parameter and an asymptotic technique for the first order matrix differential equation. Cao et al. [2] considered an exponential stabilization of the system (1.2) in case of non-equal speeds. They obtained that the 'dominant' part of the close loop system is exponentially stable. Tatar [29] proved that the system with condition $\rho G<I_{\rho}$ could be stabilized in an exponential manner using boundary controls. As the same problem in [31], their results improved the few existing similar works in the literature. Furthermore, Lo and Tatar [19] investigated uniform stability of the system

$$
\left\{\begin{array}{rr}
\rho \varphi_{t t}+G\left(\psi-\varphi_{x}\right)_{x}=0, & (x, t) \in(0,1) \times(0,+\infty), \\
I_{\rho}(3 \omega-\psi)_{t t}-(3 \omega-\psi)_{x x}-G\left(\psi-\varphi_{x}\right) & (x, t) \in(0,1) \times(0,+\infty), \\
\quad+\int_{0}^{t} h(t-\tau)(3 \omega-\psi)_{x x}(\tau) d \tau=0, & (x, t) \in(0,1) \times(0,+\infty), \\
I_{\rho} \omega_{t t}-\omega_{x x}+G\left(\psi-\varphi_{x}\right)+\frac{4}{3} \gamma \omega+\int_{0}^{t} g(t-\tau) \omega_{x x}(\tau) d \tau=0, & (0.6)
\end{array}\right.
$$


when a viscoelastic damping acted on the effective rotation and in the slip. This extended previous works where boundary controls were used in addition to a frictional damping in the dynamic of the slip. For other asymptotic behavior results to laminated beams, we refer the reader to $[1,15,18,20]$ and the references therein.

It is easy to find that if the slip $\omega$ is assumed to be identically zero, then the first two equations of system (1.2) can be reduced exactly to the Timoshenko beam system. For Timoshenko system in thermoelasticity of type III, the theory of which was proposed by Green and Naghdi [8], a large number of interesting decay results depending on the stability number have been established (see [6, 21, 22, 24, 27] and references therein). Messaoudi and Said-Houari [23] considered the following one-dimensional linear thermoelastic system:

$$
\begin{cases}\rho_{1} \varphi_{t t}-K\left(\varphi_{x}+\psi\right)_{x}=0, & (x, t) \in(0,1) \times(0,+\infty), \\ \rho_{2} \psi_{t t}-b \psi_{x x}-K\left(\varphi_{x}+\psi\right)+\beta \theta_{x}=0, & (x, t) \in(0,1) \times(0,+\infty), \\ \rho_{3} \theta_{t t}-\delta \theta_{x x}+\gamma \psi_{t t x}-k \theta_{t x x}=0, & (x, t) \in(0,1) \times(0,+\infty),\end{cases}
$$

which modeled the transverse vibration of a thick beam with heat conduction. Under the condition $\frac{k}{\rho_{1}}=\frac{b}{\rho_{2}}$, they proved that weak solution decay exponentially by using the energy method. Moreover, Kafini et al. [13] studied the following Timoshenko system of thermoelasticity of type III with delay of the form:

$$
\left\{\begin{array}{lr}
\rho_{1} \phi_{t t}-K\left(\phi_{x}+\psi\right)_{x}+\mu_{1} \phi_{t}(x, t)+\mu_{2} \phi_{t}(x, t-\tau)=0, & (x, t) \in(0,1) \times(0,+\infty), \\
\rho_{2} \psi_{t t}-b \psi_{x x}+K\left(\phi_{x}+\psi\right)+\beta \theta_{t x}=0, & (x, t) \in(0,1) \times(0,+\infty), \\
\rho_{3} \theta_{t t}-\delta \theta_{x x}+\gamma \psi_{t x}-k \theta_{t x x}=0, & (x, t) \in(0,1) \times(0,+\infty) .
\end{array}\right.
$$

Under the initial and boundary conditions

$$
\begin{cases}\theta(\cdot, 0)=\theta_{0}, \theta_{t}(\cdot, 0)=\theta_{1}, \psi(\cdot, 0)=\psi_{0}, & x \in[0,1], \\ \psi_{t}(\cdot, 0)=\psi_{1}, \phi(\cdot, 0)=\phi_{0}, \phi_{t}(\cdot, 0)=\phi_{1}, & x \in[0,1], \\ \phi_{t}(x, t-\tau)=f_{0}(x, t-\tau), & t \in(0, \tau), \\ \phi(0, t)=\phi(1, t)=\psi(0, t)=\psi(1, t)=\theta_{x}(0, t)=\theta_{x}(1, t)=0, t \in[0,+\infty),\end{cases}
$$

the energy of system decays exponentially in the case of equal wave speeds and polynomially in the case of nonequal wave speeds. For other related results, we refer the reader to $[3,4,5,11$, $12,16,17,26,28]$.

Motivated by the above results, in the present work, we study the well-posedness and asymptotic behaviour of solutions to the laminated beam (1.1) in thermoelasticity of type III. By using semigroup method and Lumer-Philips theorem, we prove the existence and uniqueness of the solution. By using the perturbed energy method and construct some Lyapunov functionals, we then obtain the exponential decay result for the case of equal wave speeds, i.e., $\frac{G}{\rho_{1}}=\frac{D}{I_{\rho_{1}}}$. When $\frac{G}{\rho_{1}} \neq \frac{D}{I_{\rho_{1}}}$, we obtain the lack of exponential stability by using Gearhart-Herbst-Prüss-Huang theorem. For this case, by introducing the extra second-order energy, we prove the polynomial decay result. 
The rest of our paper is organized as follows. In the next section, we introduce some preliminaries and state the main results. In Section 3, we establish the well-posedness of the system. In Section 4, we prove that the system is exponentially stable in the case of equal wave speeds. In Section 5, we show that the system is lack of exponential stability with different wave-propagation speeds. The proof of the polynomial decay result is given in Section 6 .

\section{Preliminaries and main results}

To exhibit the dissipative nature of the system (1.1), we introduce some new variables

$$
\Phi=\varphi_{t}, \Psi=\psi_{t}, W=\omega_{t}
$$

Then system (1.1) takes the form:

$$
\begin{cases}\rho_{1} \Phi_{t t}+G\left(\Psi-\Phi_{x}\right)_{x}=0, & (x, t) \in(0,1) \times(0,+\infty), \\ I_{\rho_{1}}(3 W-\Psi)_{t t}-D(3 W-\Psi)_{x x}-G\left(\Psi-\Phi_{x}\right)+\alpha \theta_{t x}=0, & (x, t) \in(0,1) \times(0,+\infty), \\ I_{\rho_{1}} W_{t t}-D W_{x x}+G\left(\Psi-\Phi_{x}\right)+\frac{4}{3} \beta_{1} W+\frac{4}{3} \beta_{2} W_{t}=0, & (x, t) \in(0,1) \times(0,+\infty), \\ \rho_{2} \theta_{t t}-\delta \theta_{x x}+\gamma(3 W-\Psi)_{t x}-k \theta_{t x x}=0, & (x, t) \in(0,1) \times(0,+\infty),\end{cases}
$$

with the initial data and boundary conditions

$$
\begin{cases}\Phi(x, 0)=\Phi_{0}(x), \Psi(x, 0)=\Psi_{0}(x), & x \in[0,1], \\ W(x, 0)=W_{0}(x), \theta(x, 0)=\theta_{0}(x), & x \in[0,1], \\ \Phi_{t}(x, 0)=\Phi_{1}(x), \Psi_{t}(x, 0)=\Psi_{1}(x), & x \in[0,1], \\ W_{t}(x, 0)=W_{1}(x), \theta_{t}(x, 0)=\theta_{1}(x), & x \in[0,1], \\ \Phi_{x}(0, t)=\Phi_{x}(1, t)=\Psi(0, t)=\Psi(1, t)=0, & t \in[0,+\infty), \\ W(0, t)=W(1, t)=\theta_{x}(0, t)=\theta_{x}(1, t)=0, & t \in[0,+\infty)\end{cases}
$$

where

$$
\begin{cases}\Phi_{0}(x)=\varphi_{1}, \Phi_{1}(x)=-\frac{G}{\rho_{1}}\left(\psi_{0}-\varphi_{0 x}\right)_{x}, \Psi_{0}(x)=\psi_{1}, & x \in[0,1], \\ \Psi_{1}(x)=-\frac{4 G}{I_{\rho_{1}}}\left(\psi_{0}-\phi_{0 x}\right)-\frac{D}{I_{\rho_{1}}}\left(3 \omega_{0}-\psi_{0}\right)_{x x}+\frac{\alpha}{I_{\rho_{1}}} \theta_{1 x}-\frac{4 \beta_{1}}{I_{\rho_{1}}} \omega_{0}-\frac{4 \beta_{2}}{I_{\rho_{1}}} \omega_{1}+\frac{3 D}{I_{\rho_{1}}} \omega_{0 x x}, & x \in[0,1], \\ W_{0}(x)=\omega_{1}, W_{1}(x)=-\frac{G}{I_{\rho_{1}}}\left(\psi_{0}-\varphi_{0 x}\right)-\frac{4 \beta_{1}}{3 I \rho_{1}} \omega_{0}-\frac{4 \beta_{1}}{3 I_{\rho_{1}}} \omega_{1}+\frac{D}{I_{\rho_{1}}} \omega_{0 x x}, & x \in[0,1] .\end{cases}
$$

From equations $(2.1)_{1},(2.1)_{3}$ and $(2.2)$, we easily verify that

$$
\frac{d^{2}}{d t^{2}} \int_{0}^{1} \Phi(x, t) d x=0, \frac{d^{2}}{d t^{2}} \int_{0}^{1} \theta(x, t) d x=0,
$$

and

$$
\bar{\Phi}(x, t):=\Phi(x, t)-\int_{0}^{1} \Phi_{0}(x) d x-t \int_{0}^{1} \Phi_{1}(x) d x,
$$




$$
\bar{\theta}(x, t):=\theta(x, t)-\int_{0}^{1} \theta_{0}(x) d x-t \int_{0}^{1} \theta_{1}(x) d x .
$$

We know that $(\bar{\Phi}, \Psi, W, \bar{\theta})$ satisfies the boundary conditions, and more importantly

$$
\int_{0}^{1} \bar{\Phi}(x, t) d x=0, \int_{0}^{1} \bar{\theta}(x, t) d x=0 .
$$

Hence, the use of Poincaré's inequality for $\bar{\Phi}$ and $\bar{\theta}$ is justified. In what follows, we will work with $\bar{\Phi}$ and $\bar{\theta}$. For convenience, we write $\Phi$ and $\theta$.

From now on, we let

$$
U=\left(\Phi, 3 W-\Psi, W, \theta, \Phi_{t}, 3 W_{t}-\Psi_{t}, W_{t}, \theta_{t}\right)
$$

then (2.1) and (2.2) can be written as an evolutionary equation

$$
\left\{\begin{array}{l}
\frac{d U(t)}{d t}=\mathcal{A} U(t), \quad t>0 \\
U(0)=U_{0}=\left(\Phi_{0}, 3 W_{0}-\Psi_{0}, W_{0}, \theta_{0}, \Phi_{1}, 3 W_{1}-\Psi_{1}, W_{1}, \theta_{1}\right),
\end{array}\right.
$$

where $\mathcal{A}$ is a linear operator defined by

$$
\mathcal{A U}=\left(\begin{array}{c}
\Phi_{t} \\
3 W_{t}-\Psi_{t} \\
W_{t} \\
\theta_{t} \\
-\frac{G}{\rho_{1}}\left(\Psi-\Phi_{x}\right)_{x} \\
\frac{G}{I_{\rho_{1}}}\left(\Psi-\Phi_{x}\right)+\frac{D}{I_{\rho_{1}}}(3 W-\Psi)_{x x}-\frac{\alpha}{I_{\rho_{1}}} \theta_{x t} \\
-\frac{4 \beta_{1}}{I_{\rho_{1}}}\left(\Psi-\Phi_{x}\right)-\frac{4 \beta_{2}}{3 I_{\rho_{1}}} W_{t}+\frac{D}{I_{\rho_{1}}} W_{x x} \\
\frac{\delta}{\rho_{2}} \theta_{x x}-\frac{\gamma}{\rho_{2}}(3 W-\Psi)_{t x}+\frac{k}{\rho_{2}} \theta_{t x x}
\end{array}\right) .
$$

We consider the following spaces:

$$
\begin{aligned}
& L_{*}^{2}(0,1)=\left\{w \in L^{2}(0,1): \int_{0}^{1} w(s) d s=0\right\}, \\
& H_{*}^{1}(0,1)=H^{1}(0,1) \cap L_{*}^{2}(0,1), \\
& H_{*}^{2}(0,1)=\left\{w \in H^{2}(0,1): w_{x}(0)=w_{x}(1)=0\right\},
\end{aligned}
$$

and the energy space:

$$
\mathcal{H}=H_{*}^{1}(0,1) \times H_{0}^{1}(0,1) \times H_{0}^{1}(0,1) \times H_{*}^{1}(0,1) \times L_{*}^{2}(0,1) \times L^{2}(0,1) \times L^{2}(0,1) \times L_{*}^{2}(0,1)
$$

The inner product on Hilbert space $\mathcal{H}$ is defined by

$$
(U, \bar{U})_{\mathcal{H}}=\gamma \rho_{1} \int_{0}^{1} \Phi_{t} \bar{\Phi}_{t} d x+\gamma G \int_{0}^{1}\left(\Psi-\Phi_{x}\right)\left(\bar{\Psi}-\bar{\Phi}_{x}\right) d x+\gamma I_{\rho_{1}} \int_{0}^{1}(3 W-\Psi)_{t}(3 \bar{W}-\bar{\Psi})_{t} d x
$$




$$
\begin{aligned}
& +\gamma \int_{0}^{1} D(3 W-\Psi)_{x}(3 \bar{W}-\bar{\Psi})_{x} d x+3 \gamma \int_{0}^{1} I_{\rho_{1}} W_{t} \bar{W}_{t} d x+4 \gamma \beta_{1} \int_{0}^{1} W \bar{W} d x \\
& +3 \gamma D \int_{0}^{1} W_{x} \bar{W}_{x} d x+\alpha \rho_{2} \int_{0}^{1} \theta_{t} \bar{\theta}_{t} d x+\alpha \delta \int_{0}^{1} \theta_{x} \bar{\theta}_{x} d x
\end{aligned}
$$

The domain of $\mathcal{A}$ is

$$
D(\mathcal{A})=\left\{\begin{array}{c}
U \in \mathcal{H} \mid \Phi, \theta \in H_{*}^{2}(0,1) \cap H_{*}^{1}(0,1), \Psi, W \in H^{2}(0,1) \cap H_{0}^{1}(0,1), \\
\Phi_{t}, \theta_{t} \in H_{*}^{1}(0,1), \Psi_{t}, W_{t} \in H_{0}^{1}(0,1), \delta \theta+k \theta_{t} \in H_{*}^{2}(0,1)
\end{array}\right\}
$$

and it is dense in $\mathcal{H}$.

We give the following well-posedness result of problem (2.3) :

Theorem 2.1 Let $U_{0} \in \mathcal{H}$, then problem (2.3) exists a unique solution $U \in C\left(\mathbb{R}^{+}, \mathcal{H}\right)$. Moreover, if $U_{0} \in D(\mathcal{A})$ then $U \in C\left(\mathbb{R}^{+}, D(\mathcal{A})\right) \cap C^{1}\left(\mathbb{R}^{+}, \mathcal{H}\right)$.

To state our decay result, we introduce the first energy functional

$$
\begin{aligned}
E(t)= & \frac{1}{2} \int_{0}^{1}\left[\gamma \rho_{1} \Phi_{t}^{2}+\gamma G\left(\Psi-\Phi_{x}\right)^{2}+\gamma I_{\rho_{1}}(3 W-\Psi)_{t}^{2}+\gamma D(3 W-\Psi)_{x}^{2}\right] d x \\
& +\frac{1}{2} \int_{0}^{1}\left[3 \gamma I_{\rho_{1}} W_{t}^{2}+4 \gamma \beta_{1} W^{2}+3 \gamma D W_{x}^{2}+\alpha \rho_{2} \theta_{t}^{2}+\alpha \delta \theta_{x}^{2}\right] d x
\end{aligned}
$$

and the second-order energy functional

$$
\begin{aligned}
E_{2}(t)= & \frac{1}{2} \int_{0}^{1}\left[\gamma \rho_{1} \Phi_{t t}^{2}+\gamma G\left(\Psi-\Phi_{x}\right)_{t}^{2}+\gamma I_{\rho_{1}}(3 W-\Psi)_{t t}^{2}+\gamma D(3 W-\Psi)_{x t}^{2}\right] d x \\
& +\frac{1}{2} \int_{0}^{1}\left[3 \gamma I_{\rho_{1}} W_{t t}^{2}+4 \gamma \beta_{1} W_{t}^{2}+3 \gamma D W_{x t}^{2}+\alpha \rho_{2} \theta_{t t}^{2}+\alpha \delta \theta_{x t}^{2}\right] d x
\end{aligned}
$$

Our decay results state as follows.

Theorem 2.2 Assume that $\frac{G}{\rho_{1}}=\frac{D}{I_{\rho_{1}}}$ and $U_{0} \in \mathcal{H}$. Then, there exist two positive constants $C_{0}$ and $s$, such that the energy $E(t)$ associated with problem (2.1)-(2.2) satisfies

$$
E(t) \leq C_{0} E(0) e^{-s t}, \quad t \geq 0 .
$$

Theorem 2.3 Assume that $\frac{G}{\rho_{1}} \neq \frac{D}{I_{\rho_{1}}}$ and $U_{0} \in \mathcal{H}$. Then the semigroup associated to system (2.1) with boundary conditions (2.2) is not exponentially stable.

Theorem 2.4 Assume that $\frac{G}{\rho_{1}} \neq \frac{D}{I_{\rho_{1}}}$ and $U_{0} \in \mathcal{H}$. Then, there exists a positive constant $C_{1}$ such that the energy $E(t)$ associated with problem (2.1)-(2.2) satisfies

$$
E(t) \leq \frac{C_{1}\left(E(0)+E_{2}(0)\right)}{t}, t \geq 0
$$

To study the property of system (2.1), we need the following inequality.

Lemma 2.1 [30, Lemma 2.2] Let $f \in\left\{H^{1}[0,1] \mid f(0)=0\right\}$, then it holds that

$$
\int_{0}^{1} f^{2}(x) d x \leq \frac{1}{2} \int_{0}^{1}\left(f^{\prime}(x)\right)^{2} d x .
$$




\section{Well-posedness}

In this section, we give the proof of the well-posedness of problem (2.1)-(2.2) by making use of Lumer-Philips theorem [7, 25].

Proof of Theorem 2.1. To prove the well-posedness result, it suffices to show that $\mathcal{A}$ : $D(\mathcal{A}) \rightarrow \mathcal{H}$ is a maximal monotone operator, which means $\mathcal{A}$ is dissipative and $I d-\mathcal{A}$ is surjective. First, an easy computation implies that

$$
\begin{aligned}
(\mathcal{A} U, U)_{\mathcal{H}}= & \gamma \rho_{1} \int_{0}^{1} \Phi_{t t} \Phi_{t} d x+\gamma G \int_{0}^{1}\left(\Psi-\Phi_{x}\right)_{t}\left(\Psi-\Phi_{x}\right) d x+\gamma I_{\rho_{1}} \int_{0}^{1}(3 W-\Psi)_{t t}(3 W-\Psi)_{t} d x \\
& +\gamma \int_{0}^{1} D(3 W-\Psi)_{t x}(3 W-\Psi)_{x} d x+3 \gamma \int_{0}^{1} I_{\rho_{1}} W_{t t} W_{t} d x+4 \gamma \beta_{1} \int_{0}^{1} W_{t} W d x \\
& +3 \gamma D \int_{0}^{1} W_{t x} W_{x} d x+\alpha \rho_{2} \int_{0}^{1} \theta_{t t} \theta_{t} d x+\alpha \delta \int_{0}^{1} \theta_{t x} \theta_{x} d x
\end{aligned}
$$

For any $U \in D(\mathcal{A})$, we have

$$
(\mathcal{A} U, U)_{\mathcal{H}}=-4 \gamma \int_{0}^{1} \beta_{2} W_{t}^{2} d x-\alpha \int_{0}^{1} k \theta_{t x}^{2} d x \leq 0 .
$$

Consequently, $\mathcal{A}$ is a dissipative operator.

Next, let $F=\left(f_{1}, \cdots, f_{8}\right)^{T} \in \mathcal{H}$, we seek $V=\left(v_{1}, \cdots, v_{8}\right)^{T} \in D(\mathcal{A})$ satisfying

$$
(I d-\mathcal{A}) V=F,
$$

that is

$$
\left\{\begin{array}{l}
v_{1}-v_{5}=f_{1}, \\
v_{2}-v_{6}=f_{2}, \\
v_{3}-v_{7}=f_{3}, \\
v_{4}-v_{8}=f_{4}, \\
\rho_{1} v_{5}-G \partial_{x x} v_{1}-G \partial_{x} v_{2}+3 G \partial_{x} v_{3}=\rho_{1} f_{5}, \\
I_{\rho_{1}} v_{6}+G \partial_{x} v_{1}+G v_{2}-3 G v_{3}-D \partial_{x x} v_{2}+\alpha \partial_{x} v_{8}=I_{\rho_{1}} f_{6}, \\
I_{\rho_{1}} v_{7}-G v_{2}+3 G v_{3}-G \partial_{x} v_{1}+\frac{4}{3} \beta_{1} v_{3}+\frac{4}{3} \beta_{2} v_{7}-D \partial_{x x} v_{3}=I_{\rho_{1}} f_{7}, \\
\rho_{2} v_{8}-\delta \partial_{x x} v_{4}+\gamma \partial_{x} v_{6}-k \partial_{x x} v_{8}=\rho_{2} f_{8} .
\end{array}\right.
$$

From $(3.2)_{1}-(3.2)_{4}$, we have

$$
\left\{\begin{array}{l}
v_{5}=v_{1}-f_{1}, \\
v_{6}=v_{2}-f_{2}, \\
v_{7}=v_{3}-f_{3}, \\
v_{8}=v_{4}-f_{4} .
\end{array}\right.
$$


By combining (3.2) and (3.3), it can be shown that $v_{1}, v_{2}, v_{3}, v_{4}$ satisfy

$$
\left\{\begin{array}{l}
\rho_{1} v_{1}-G \partial_{x x} v_{1}-G \partial_{x} v_{2}+3 G \partial_{x} v_{3}=\rho_{1}\left(f_{1}+f_{5}\right), \\
I_{\rho_{1}} v_{2}+G \partial_{x} v_{1}+G v_{2}-3 G v_{3}-D \partial_{x x} v_{2}+\alpha \partial_{x} v_{4}=I_{\rho_{1}}\left(f_{2}+f_{6}\right)+\alpha \partial_{x} f_{4} \\
I_{\rho_{1}} v_{3}-G v_{2}+3 G v_{3}-G \partial_{x} v_{1}+\frac{4}{3} \beta_{1} v_{3}+\frac{4}{3} \beta_{2} v_{3}-D \partial_{x x} v_{3}=I_{\rho_{1}}\left(f_{3}+f_{7}\right)+\frac{4}{3} \beta_{2} f_{3}, \\
\rho_{2} v_{4}-\delta \partial_{x x} v_{4}+\gamma \partial_{x} v_{2}-k \partial_{x x} v_{4}=\rho_{2}\left(f_{4}+f_{8}\right)+\gamma \partial_{x} f_{2}-k \partial_{x x} f_{4} .
\end{array}\right.
$$

Multiplying $(3.4)_{1}-(3.4)_{4}$ by $\gamma \bar{v}_{1}, \gamma \bar{v}_{2}, 3 \gamma \bar{v}_{3}$ and $\alpha \bar{v}_{4}$ and integrating over $(0,1)$, we arrive at

$$
\begin{aligned}
& \gamma \int_{0}^{1} \rho_{1} v_{1} \bar{v}_{1} d x-\gamma \int_{0}^{1} G \partial_{x x} v_{1} \bar{v}_{1} d x-\gamma \int_{0}^{1} G \partial_{x} v_{2} \bar{v}_{1} d x+3 \gamma \int_{0}^{1} G \partial_{x} v_{3} \bar{v}_{1} d x \\
& =\gamma \int_{0}^{1} \rho_{1}\left(f_{1}+f_{5}\right) \bar{v}_{1} d x \\
& \gamma \int_{0}^{1} I_{\rho_{1}} v_{2} \bar{v}_{2} d x+\gamma \int_{0}^{1} G \partial_{x} v_{1} \bar{v}_{2} d x+\gamma \int_{0}^{1} G v_{2} \bar{v}_{2} d x-3 \gamma \int_{0}^{1} G v_{3} \bar{v}_{2} d x-\gamma \int_{0}^{1} D \partial_{x x} v_{2} \bar{v}_{2} d x \\
& +\gamma \int_{0}^{1} \alpha \partial_{x} v_{4} \bar{v}_{2} d x=\gamma \int_{0}^{1} I_{\rho_{1}}\left(f_{2}+f_{6}\right) \bar{v}_{2} d x+\gamma \int_{0}^{1} \alpha \partial_{x} f_{4} \bar{v}_{2} d x \\
& 3 \gamma \int_{0}^{1} I_{\rho_{1}} v_{3} \bar{v}_{3} d x-3 \gamma \int_{0}^{1} G v_{2} \bar{v}_{3} d x+9 \gamma \int_{0}^{1} G v_{3} \bar{v}_{3} d x-3 \gamma \int_{0}^{1} G \partial_{x} v_{1} \bar{v}_{3} d x+4 \gamma \int_{0}^{1} \beta_{1} v_{3} \bar{v}_{3} d x \\
& +4 \gamma \int_{0}^{1} \beta_{2} v_{3} \bar{v}_{3} d x-3 \gamma \int_{0}^{1} D \partial_{x x} v_{3} \bar{v}_{3} d x=3 \gamma \int_{0}^{1} I_{\rho_{1}}\left(f_{3}+f_{7}\right) \bar{v}_{3} d x+4 \gamma \int_{0}^{1} \beta_{2} f_{3} \bar{v}_{3} d x \\
& \alpha \int_{0}^{1} \rho_{2} v_{4} \bar{v}_{4} d x-\alpha \int_{0}^{1} \delta \partial_{x x} v_{4} \bar{v}_{4} d x+\alpha \int_{0}^{1} \gamma \partial_{x} v_{2} \bar{v}_{4} d x-\alpha \int_{0}^{1} k \partial_{x x} v_{4} \bar{v}_{4} d x \\
& =\alpha \int_{0}^{1} \rho_{2}\left(f_{4}+f_{8}\right) \bar{v}_{4} d x+\alpha \int_{0}^{1} \gamma \partial_{x} f_{2} \bar{v}_{4} d x-\alpha \int_{0}^{1} k \partial_{x x} f_{4} \bar{v}_{4} d x .
\end{aligned}
$$

The sum of these equations in (3.5) gives the following variational formulation:

$$
\begin{array}{r}
a\left(\left(v_{1}, v_{2}, v_{3}, v_{4}\right)^{T},\left(\bar{v}_{1}, \bar{v}_{2}, \bar{v}_{3}, \bar{v}_{4}\right)^{T}\right)=\bar{a}\left(\left(\bar{v}_{1}, \bar{v}_{2}, \bar{v}_{3}, \bar{v}_{4}\right)^{T}\right), \\
\forall\left(\bar{v}_{1}, \bar{v}_{2}, \bar{v}_{3}, \bar{v}_{4}\right)^{T} \in H_{*}^{1}(0,1) \times H_{0}^{1}(0,1) \times H_{0}^{1}(0,1) \times H_{*}^{1}(0,1),
\end{array}
$$

where

$$
\begin{aligned}
& a\left(\left(v_{1}, v_{2}, v_{3}, v_{4}\right)^{T},\left(\bar{v}_{1}, \bar{v}_{2}, \bar{v}_{3}, \bar{v}_{4}\right)^{T}\right) \\
= & \gamma \int_{0}^{1} G\left(-\partial_{x} v_{1}-v_{2}+3 v_{3}\right)\left(-\partial_{x} \bar{v}_{1}-\bar{v}_{2}+3 \bar{v}_{3}\right) d x+\gamma \int_{0}^{1} \rho_{1} v_{1} \bar{v}_{1} d x \\
& +\gamma \int_{0}^{1} I_{\rho_{1}} v_{2} \bar{v}_{2} d x+\left(3 \gamma I_{\rho_{1}}+4 \gamma \beta_{1}+4 \gamma \beta_{2}\right) \int_{0}^{1} v_{3} \bar{v}_{3} d x \\
& +\alpha \int_{0}^{1} \rho_{2} v_{4} \bar{v}_{4} d x+\gamma \int_{0}^{1} D \partial_{x} v_{2} \partial_{x} \bar{v}_{2} d x+3 \gamma \int_{0}^{1} D \partial_{x} v_{3} \partial_{x} \bar{v}_{3} d x \\
& +\alpha \int_{0}^{1} \delta \partial_{x} v_{4} \partial_{x} \bar{v}_{4} d x+\alpha \int_{0}^{1} k \partial_{x} v_{4} \partial_{x} \bar{v}_{4} d x \\
& +\gamma \alpha \int_{0}^{1} \partial_{x} v_{4} \bar{v}_{2} d x+\alpha \gamma \int_{0}^{1} \partial_{x} v_{2} \bar{v}_{4} d x
\end{aligned}
$$


and

$$
\begin{aligned}
\bar{a}\left(\left(\bar{v}_{1}, \bar{v}_{2}, \bar{v}_{3}, \bar{v}_{4}\right)^{T}\right)= & \gamma \int_{0}^{1} \rho_{1}\left(f_{1}+f_{5}\right) \bar{v}_{1} d x+\gamma \int_{0}^{1} I_{\rho_{1}}\left(f_{2}+f_{6}\right) \bar{v}_{2} d x \\
& +3 \gamma \int_{0}^{1} I_{\rho_{1}}\left(f_{3}+f_{7}\right) \bar{v}_{3} d x+4 \gamma \int_{0}^{1} \beta_{2} f_{3} \bar{v}_{3} d x+\alpha \int_{0}^{1} \rho_{2}\left(f_{4}+f_{8}\right) \bar{v}_{4} d x \\
& +\alpha \gamma \int_{0}^{1} \partial_{x} f_{2} \bar{v}_{4} d x+\alpha k \int_{0}^{1} \partial_{x} f_{4} \partial_{x} \bar{v}_{4} d x+\alpha \gamma \int_{0}^{1} \partial_{x} f_{4} \bar{v}_{2} d x
\end{aligned}
$$

To study the variational formulation, we introduce the Hilbert space $V=H_{*}^{1}(0,1) \times$ $H_{0}^{1}(0,1) \times H_{0}^{1}(0,1) \times H_{*}^{1}(0,1)$ equipped with the norm

$$
\left\|\left(v_{1}, v_{2}, v_{3}, v_{4}\right)\right\|_{V}^{2}=\left\|-\partial_{x} v_{1}-v_{2}+3 v_{3}\right\|_{2}^{2}+\left\|v_{1}\right\|_{2}^{2}+\left\|\partial_{x} v_{2}\right\|_{2}^{2}+\left\|\partial_{x} v_{3}\right\|_{2}^{2}+\left\|v_{4}\right\|_{2}^{2}+\left\|\partial_{x} v_{4}\right\|_{2}^{2} .
$$

It is clear that $a(\cdot, \cdot)$ and $\bar{a}(\cdot)$ are bounded. Furthermore, we obtain

$$
\begin{aligned}
a\left(\left(v_{1}, v_{2}, v_{3}, v_{4}\right)^{T},\left(v_{1}, v_{2}, v_{3}, v_{4}\right)^{T}\right)= & \gamma \int_{0}^{1} G\left(-\partial_{x} v_{1}-v_{2}+3 v_{3}\right)^{2} d x+\gamma \int_{0}^{1} \rho_{1} v_{1}^{2} d x+\gamma \int_{0}^{1} I_{\rho_{1}} v_{2}^{2} d x \\
& +\left(3 \gamma I_{\rho_{1}}+4 \gamma \beta_{1}+4 \gamma \beta_{2}\right) \int_{0}^{1} v_{3}^{2} d x+\alpha \int_{0}^{1} \rho_{2} v_{4}^{2} d x \\
& +\gamma \int_{0}^{1} D\left(\partial_{x} v_{2}\right)^{2} d x+3 \gamma \int_{0}^{1} D\left(\partial_{x} v_{3}\right)^{2} d x \\
& +\alpha \int_{0}^{1} \delta\left(\partial_{x} v_{4}\right)^{2} d x+\alpha \int_{0}^{1} k\left(\partial_{x} v_{4}\right)^{2} d x \\
\leq & C\left\|\left(v_{1}, v_{2}, v_{3}, v_{4}\right)\right\|_{V}^{2},
\end{aligned}
$$

which implies that $a(\cdot, \cdot)$ is coercive. Consequently, applying Lax-Milgram theorem, we obtain that (3.5) has a unique solution $\left(v_{1}, v_{2}, v_{3}, v_{4}\right)^{T} \in V$.

Substituting $v_{1}, v_{2}, v_{3}$ and $v_{4}$ into (3.3), we obtain

$$
\left(v_{5}, v_{6}, v_{7}, v_{8}\right) \in H_{*}^{1}(0,1) \times H_{0}^{1}(0,1) \times H_{0}^{1}(0,1) \times H_{*}^{1}(0,1) .
$$

It remains to show that

$$
\begin{aligned}
\left(v_{1}, v_{2}, v_{3}, v_{4}\right) \in & \left(H_{*}^{2}(0,1) \cap H_{*}^{1}(0,1)\right) \times\left(H^{2}(0,1) \cap H_{0}^{1}(0,1)\right) \times\left(H^{2}(0,1) \cap H_{0}^{1}(0,1)\right) \\
& \times\left(H_{*}^{2}(0,1) \cap H_{*}^{1}(0,1)\right) .
\end{aligned}
$$

Taking $\left(\bar{v}_{2}, \bar{v}_{3}, \bar{v}_{4}\right)=(0,0,0) \in H_{0}^{1}(0,1) \times H_{0}^{1}(0,1) \times H_{*}^{1}(0,1)$ in $(3.6)$, we get

$$
\begin{aligned}
a\left(\left(v_{1}, v_{2}, v_{3}, v_{4}\right)^{T},\left(\bar{v}_{1}, 0,0,0\right)^{T}\right) & =\gamma \int_{0}^{1} \rho_{1} v_{1} \bar{v}_{1} d x+\gamma \int_{0}^{1} G\left(-\partial_{x x} v_{1} \bar{v}_{1}-\partial_{x} v_{2} \bar{v}_{1}+3 \partial_{x} v_{3} \bar{v}_{1}\right) d x \\
& =\gamma \int_{0}^{1} \rho_{1}\left(f_{1}+f_{5}\right) \bar{v}_{1} d x
\end{aligned}
$$

for all $\bar{v}_{1} \in H_{*}^{1}(0,1)$, which implies

$$
G \partial_{x x} v_{1}=\rho_{1} v_{1}-G \partial_{x} v_{2}+3 G \partial_{x} v_{3}-\rho_{1}\left(f_{1}+f_{5}\right) \in L_{*}^{2}(0,1) .
$$


Thus, by $\mathrm{L}^{2}$ theory for the linear elliptic equations, we obtain that

$$
v_{1} \in H^{2}(0,1) \cap H_{*}^{1}(0,1) .
$$

Moreover, (3.9) is also true for any $\phi \in C^{1}[0,1] \subset H_{*}^{1}(0,1)(\phi(0)=0)$. Hence, we get

$$
\int_{0}^{1} G \partial_{x} v_{1} \partial_{x} \phi d x+\int_{0}^{1} \rho_{1} v_{1} \phi d x-\int_{0}^{1} G \partial_{x} v_{2} \phi d x+3 \int_{0}^{1} G \partial_{x} v_{3} \phi d x=\int_{0}^{1} \rho_{1}\left(f_{1}+f_{5}\right) \phi d x
$$

By using the integration by parts, we have

$$
\partial_{x} v_{1}(1) \phi(1)-\partial_{x} v_{1}(0) \phi(0)=0 \quad \forall \phi \in C^{1}[0,1], \phi(0)=0 .
$$

Therefore, $\partial_{x} v_{1}(1) \phi(1)=0$ and we deduce that $v_{1} \in H_{*}^{2}(0,1) \cap H_{*}^{1}(0,1)$. In the same way, taking $\left(\bar{v}_{1}, \bar{v}_{3}, \bar{v}_{4}\right)=(0,0,0) \in H_{*}^{1}(0,1) \times H_{0}^{1}(0,1) \times H_{*}^{1}(0,1)$ in $(3.7)$, one has

$$
\begin{aligned}
a\left(\left(v_{1}, v_{2}, v_{3}, v_{4}\right)^{T},\left(0, \bar{v}_{2}, 0,0\right)^{T}\right)= & \gamma \int_{0}^{1} G\left(\partial_{x} v_{1} \bar{v}_{2}+v_{2} \bar{v}_{2}-3 v_{3} \bar{v}_{2}\right) d x+\gamma \int_{0}^{1} I_{\rho_{1}} v_{2} \bar{v}_{2} d x \\
& +\gamma \int_{0}^{1} D \partial_{x} v_{2} \partial_{x} \bar{v}_{2} d x+\gamma \alpha \int_{0}^{1} \partial_{x} v_{4} \bar{v}_{2} d x \\
= & \gamma \int_{0}^{1} I_{\rho_{1}}\left(f_{2}+f_{6}\right) \bar{v}_{2} d x+\alpha \gamma \int_{0}^{1} \partial_{x} f_{4} \bar{v}_{2} d x .
\end{aligned}
$$

Recalling $(3.2)_{2}$ and $(3.2)_{4}$, we arrive at

$$
\begin{aligned}
& \int_{0}^{1} G\left(\partial_{x} v_{1} \bar{v}_{2}+v_{2} \bar{v}_{2}-3 v_{3} \bar{v}_{2}\right) d x+\int_{0}^{1} I_{\rho_{1}} v_{2} \bar{v}_{2} d x+\int_{0}^{1} D \partial_{x} v_{2} \partial_{x} \bar{v}_{2} d x+\alpha \int_{0}^{1} \partial_{x} v_{4} \bar{v}_{2} d x \\
= & \int_{0}^{1} I_{\rho_{1}}\left(v_{2}-v_{6}+f_{6}\right) \bar{v}_{2} d x+\alpha \int_{0}^{1} \partial_{x}\left(v_{4}-v_{8}\right) \bar{v}_{2} d x .
\end{aligned}
$$

Hence, one has

$$
\int_{0}^{1} D \partial_{x} v_{2} \partial_{x} \bar{v}_{2} d x=\int_{0}^{1}\left[I_{\rho_{1}} f_{6}-G\left(\partial_{x} v_{1}+v_{2}-3 v_{3}\right)-\alpha \partial_{x} v_{8}-I_{\rho_{1}} v_{6}\right] \bar{v}_{2} d x
$$

for all $\bar{v}_{2} \in H_{0}^{1}(0,1)$, which implies $I_{\rho_{1}} f_{6}-G\left(\partial_{x} v_{1}+v_{2}-3 v_{3}\right)-\alpha \partial_{x} v_{8}-I_{\rho_{1}} v_{6} \in L^{2}(0,1)$. Consequently, (3.11) takes the form

$$
\int_{0}^{1}\left(-D \partial_{x x} v_{2}+G \partial_{x} v_{1}+G v_{2}-3 G v_{3}+\alpha \partial_{x} v_{8}+I_{\rho_{1}} v_{6}-I_{\rho_{1}} f_{6}\right) \bar{v}_{2} d x=0
$$

We obtain

$$
-D \partial_{x x} v_{2}+G\left(\partial_{x} v_{1}+v_{2}-3 v_{3}\right)+\alpha \partial_{x} v_{8}+I_{\rho_{1}} v_{6}=I_{\rho_{1}} f_{6},
$$

and $v_{2} \in H^{2}(0,1) \cap H_{0}^{1}(0,1)$, which gives $(3.2)_{6}$. Similarly, we get

$$
v_{3} \in H^{2}(0,1) \cap H_{0}^{1}(0,1) .
$$

However, if we take $\left(\bar{v}_{1}, \bar{v}_{2}, \bar{v}_{3}\right)=(0,0,0) \in H_{*}^{1}(0,1) \times H_{0}^{1}(0,1) \times H_{0}^{1}(0,1)$ in $(3.7)$, thanks to $(3.2)_{2}$ and $(3.2)_{4}$, we get

$$
\delta \partial_{x x} v_{4}+k \partial_{x x} v_{8}=\rho_{2} f_{8}-\gamma \partial_{x} v_{6}-\rho_{2} v_{8} \in L_{*}^{2}(0,1),
$$


and conclude that

$$
\delta v_{4}+k v_{8} \in H^{2}(0,1)
$$

Furthermore, it is obvious from

$$
\delta \partial_{x} v_{4}+k \partial_{x} v_{8}=\rho_{2} \int_{0}^{x} f_{8} d x-\gamma v_{6}-\rho_{2} \int_{0}^{x} v_{8} d x
$$

that

$$
\left(\delta \partial_{x} v_{4}+k \partial_{x} v_{8}\right)(0)=\left(\delta \partial_{x} v_{4}+k \partial_{x} v_{8}\right)(1)=0
$$

then, we get

$$
\delta v_{4}+k v_{8} \in H_{*}^{2}(0,1)
$$

Hence, there exists a unique $V \in D(\mathcal{A})$ such that (3.1) is satisfied, the operator $I d-\mathcal{A}$ is surjective. Moreover, it is easy to see that $D(\mathcal{A})$ is dense in $\mathcal{H}$. Consequently, the well-posedness result follows from Lumer-Philips theorem.

\section{Exponential decay for $\frac{G}{\rho_{1}}=\frac{D}{I_{\rho_{1}}}$}

In this section, we prove the exponential decay result in Theorem 2.2. It will be accomplished by a Lyapunov functional $\mathcal{L}(t)$ which equivalent to $E(t)$. To construct the Lyapunov functional $\mathcal{L}(t)$, we modify some classical multipliers used in [13] and prove several lemmas.

The following lemma shows that the associated energy is non-increasing in time.

Lemma 4.1 The energy functional $E(t)$ defined by (2.4) satisfies

$$
\frac{d}{d t} E(t)=-4 \gamma \int_{0}^{1} \beta_{2} W_{t}^{2} d x-\alpha \int_{0}^{1} k \theta_{t x}^{2} d x \leq 0
$$

Proof. Mulitiplying equation $(2.2)_{1}$ by $\gamma \Phi_{t},(2.2)_{2}$ by $\gamma(3 W-\Psi)_{t},(2.2)_{3}$ by $3 \gamma W_{t}$ and $(2.2)_{4}$ by $\alpha \theta_{t}$ respectively, then, integrating over $(0,1)$ and summing up, we get

$$
\begin{aligned}
& \quad \frac{d}{d t} \frac{\gamma}{2} \int_{0}^{1}\left[\rho_{1} \Phi_{t}^{2}+G\left(\Psi-\Phi_{x}\right)^{2}+I_{\rho_{1}}\left(3 W_{t}-\Psi_{t}\right)^{2}+D\left(3 W_{x}-\Psi_{x}\right)^{2}+3 I_{\rho_{1}} W_{t}^{2}+4 \beta_{1} W^{2}+3 D W_{x}^{2}\right] d x \\
& +\frac{d}{d t} \frac{\alpha}{2} \int_{0}^{1}\left(\rho_{2} \theta_{t}^{2}+\delta \theta_{x}^{2}\right) d x+4 \gamma \int_{0}^{1} \beta_{2} W_{t}^{2} d x+\alpha \int_{0}^{1} k\left(\theta_{t x}\right)^{2} d x-G \int_{0}^{1}\left(\Psi-\Phi_{x}\right) \Psi_{t} d x \\
& \quad-\gamma \int_{0}^{1} G\left(\Psi-\Phi_{x}\right)(3 W-\Psi)_{t} d x+3 \gamma \int_{0}^{1} G\left(\Psi-\Phi_{x}\right) W_{t} d x \\
& =0
\end{aligned}
$$

we can conclude that

$$
\frac{d}{d t} E(t)=-4 \gamma \int_{0}^{1} \beta_{2} W_{t}^{2} d x-\alpha \int_{0}^{1} k\left(\theta_{t x}\right)^{2} d x
$$

This completes the proof. 
Lemma 4.2 Let $(\Phi, \Psi, W, \theta)$ be the solution of problem (2.1)-(2.2). The functional

$$
I_{1}(t):=-\rho_{1} \int_{0}^{1} \Phi \Phi_{t} d x+I_{\rho_{1}} \int_{0}^{1} W W_{t} d x
$$

satisfies

$$
\begin{aligned}
I_{1}^{\prime}(t) \leq & -\rho_{1} \int_{0}^{1} \Phi_{t}^{2} d x-\frac{2 \gamma \beta}{3} \int_{0}^{1} W^{2} d x-\frac{D}{2} \int_{0}^{1} W_{x}^{2} d x+C \int_{0}^{1} W_{t}^{2} d x \\
& +C \int_{0}^{1}\left(\Psi-\Phi_{x}\right)^{2} d x+C \int_{0}^{1}\left(3 W_{x}-\Psi_{x}\right)^{2} d x .
\end{aligned}
$$

Proof. By differentiating $I_{1}$ and using $(2.1)_{1}$, we conclude that

$$
\begin{aligned}
I_{1}(t)= & -\rho_{1} \int_{0}^{1} \Phi_{t}^{2} d x-\rho_{1} \int_{0}^{1} \Phi \Phi_{t t} d x+I_{\rho_{1}} \int_{0}^{1} W_{t}^{2} d x+I_{\rho_{1}} \int_{0}^{1} W W_{t t} d x \\
= & -\rho_{1} \int_{0}^{1} \Phi_{t}^{2} d x+I_{\rho_{1}} \int_{0}^{1} W_{t}^{2} d x-G \int_{0}^{1} \Phi_{x}\left(\Psi-\Phi_{x}\right) d x-G \int_{0}^{1} W\left(\Psi-\Phi_{x}\right) d x \\
& -\frac{4 \beta_{1}}{3} \int_{0}^{1} W^{2} d x-\frac{4 \beta_{2}}{3} \int_{0}^{1} W W_{t} d x-D \int_{0}^{1} W_{x}^{2} d x .
\end{aligned}
$$

Making use of Young's inequality, we get

$$
\begin{aligned}
I_{1}^{\prime}(t) \leq & -\rho_{1} \int_{0}^{1} \Phi_{t}^{2} d x-\frac{4 \beta_{1}}{3} \int_{0}^{1} W^{2} d x-D \int_{0}^{1} W_{x}^{2} d x+I_{\rho_{1}} \int_{0}^{1} W_{t}^{2} d x+G \int_{0}^{1}\left(\Psi-\Phi_{x}\right)^{2} d x \\
& +\frac{D}{36 G} \int_{0}^{1} \Psi^{2} d x+\frac{9 G^{2}}{D} \int_{0}^{1}\left(\Psi-\Phi_{x}\right)^{2} d x+\frac{\beta_{1}}{3} \int_{0}^{1} W^{2} d x+\frac{3 G^{2}}{4 \beta_{1}} \int_{0}^{1}\left(\Psi-\Phi_{x}\right)^{2} d x \\
& +\frac{\beta_{1}}{3} \int_{0}^{1} W^{2} d x+\frac{4 \beta_{2}^{2}}{3 \beta_{1}} \int_{0}^{1} W_{t}^{2} d x .
\end{aligned}
$$

Using Lemma 2.1 and Young's inequality again, we obtain (4.1).

Lemma 4.3 Let $(\Phi, \Psi, W, \theta)$ be the solution of problem (2.1)-(2.2). The functional

$$
I_{2}(t):=I_{\rho_{1}} \int_{0}^{1}(3 W-\Psi)(3 W-\Psi)_{t} d x
$$

satisfies

$$
I_{2}^{\prime}(t) \leq-\frac{D}{2} \int_{0}^{1}\left(3 W_{x}-\Psi_{x}\right)^{2} d x+I_{\rho_{1}} \int_{0}^{1}\left(3 W_{t}-\Psi_{t}\right)^{2} d x+C \int_{0}^{1}\left(\Psi-\Phi_{x}\right)^{2} d x+C \int_{0}^{1} \theta_{t}^{2} d x .
$$

Proof. By differentiating $I_{2}$ and using $(2.1)_{2}$, we conclude that

$$
\begin{aligned}
I_{2}^{\prime}(t)= & I_{\rho_{1}} \int_{0}^{1}\left(3 W_{t}-\Psi_{t}\right)^{2} d x+\int_{0}^{1}(3 W-\Psi)\left[G\left(\Psi-\Phi_{x}\right)+D(3 W-\Psi)_{x x}-\alpha \theta_{x t}\right] d x \\
= & I_{\rho_{1}} \int_{0}^{1}\left(3 W_{t}-\Psi_{t}\right)^{2} d x+\int_{0}^{1}(3 W-\Psi) G\left(\Psi-\Phi_{x}\right) d x \\
& -D \int_{0}^{1}\left(3 W_{x}-\Psi_{x}\right)^{2} d x+\alpha \int_{0}^{1}\left(3 W_{x}-\Psi_{x}\right) \theta_{t} d x
\end{aligned}
$$

Using Young's inequality and Lemma 2.1, we obtain the result. 
Lemma 4.4 Let $(\Phi, \Psi, W, \theta)$ be the solution of problem (2.1)-(2.2). The functional

$$
I_{3}(t):=I_{\rho_{1}} \rho_{2} \int_{0}^{1}(3 W-\Psi)_{t} \int_{0}^{x} \theta_{t}(y, t) d y d x-\delta I_{\rho_{1}} \int_{0}^{1} \theta_{x}(3 W-\Psi) d x
$$

satisfies

$$
\begin{aligned}
I_{3}^{\prime}(t) \leq & -\frac{\gamma I_{\rho}}{2} \int_{0}^{1}\left(3 W_{t}-\Psi_{t}\right)^{2} d x+\alpha I_{\rho_{1}} \int_{0}^{1} \theta_{t}^{2} d x+\varepsilon_{3} I_{\rho_{1}} \int_{0}^{1} G\left(\Psi-\Phi_{x}\right)^{2} d x \\
& +c\left(\varepsilon_{3}\right) \int_{0}^{1} \theta_{t}^{2} d x+\varepsilon_{3} I_{\rho_{!}} D \int_{0}^{1}\left(3 W_{x}-\Psi_{x}\right)^{2} d x .
\end{aligned}
$$

Proof. By differentiating $I_{3}$ and using $(2.1)_{4}$, we conclude that

$$
\begin{aligned}
I_{3}^{\prime}(t)= & \int_{0}^{1} I_{\rho_{1}}(3 W-\Psi)_{t} \int_{0}^{x}\left[\delta \theta_{x x}-\gamma(3 W-\Psi)_{t x}+k \theta_{t x x}\right] d y d x \\
& +\int_{0}^{1} \rho_{2}\left[G\left(\Psi-\Phi_{x}\right)+D(3 W-\Psi)_{x x}-\alpha \theta_{x t}\right] \int_{0}^{x} \theta_{t}(y, t) d y d x \\
& -\delta I_{\rho_{1}} \int_{0}^{1} \theta_{t x}(3 W-\Psi) d x-\delta I_{\rho_{1}} \int_{0}^{1} \theta_{x}(3 W-\Psi)_{t} d x \\
= & \int_{0}^{1} I_{\rho_{1}}(3 W-\Psi)_{t}\left[\delta \theta_{x}-\gamma(3 W-\Psi)_{t}+k \theta_{t x}\right] d x+\rho_{2} \int_{0}^{1} G \Psi \int_{0}^{x} \theta_{t}(y, t) d y d x \\
& +\int_{0}^{1} \rho_{2}\left[-G \Phi+D(3 W-\Psi)_{x x}-\alpha \theta_{x t}\right] \int_{0}^{x} \theta_{t}(y, t) d y d x \\
& -\delta I_{\rho_{1}} \int_{0}^{1} \theta_{t x}(3 W-\Psi) d x-\delta I_{\rho_{1}} \int_{0}^{1} \theta_{x}(3 W-\Psi)_{t} d x \\
= & -\gamma I_{\rho_{1}} \int_{0}^{1}(3 W-\Psi)_{t}^{2} d x+I_{\rho_{1}} k \int_{0}^{1}(3 W-\Psi)_{t} \theta_{t x} d x+\rho_{2} \int_{0}^{1} G_{(}\left(\Psi-\Phi_{x}\right) \int_{0}^{x} \theta_{t}(y, t) d y d x \\
& -\delta I_{\rho_{1}} \int_{0}^{1} \theta_{t x}(3 W-\Psi) d x-\rho_{2} \int_{0}^{1} D(3 W-\Psi)_{x} \theta_{t} d x+\alpha \rho_{2} \int_{0}^{2} d x \\
& +\left.\left[\rho_{2}\left(-G \Phi+D(3 W-\Psi)_{x}-\alpha \theta_{t}\right) \int_{0}^{x} \theta_{t}(y, t) d y\right]\right|_{x=0} ^{x=1} \cdot
\end{aligned}
$$

Note that

$$
\int_{0}^{1} \theta_{t}(y, t) d y=\frac{d}{d t} \int_{0}^{1} \theta(y, t) d y=0
$$

then, by Young's inequality, we obtain the result.

Lemma 4.5 Let $(\Phi, \Psi, W, \theta)$ be the solution of problem (2.1)-(2.2). The functional

$$
I_{4}(t):=\int_{0}^{1}\left[\rho_{2} \theta_{t} \theta+\frac{k}{2} \theta_{x}^{2}+\gamma(3 W-\Psi)_{x} \theta\right] d x
$$

satisfies

$$
I_{4}^{\prime}(t) \leq-\delta \int_{0}^{1} \theta_{x}^{2} d x+\left(\frac{\gamma^{2}}{4 \varepsilon_{4}}+\rho_{2}\right) \int_{0}^{1} \theta_{t}^{2} d x+\varepsilon_{4} \int_{0}^{1}\left(3 W_{x}-\Psi_{x}\right)^{2} d x
$$

Proof. By differentiating $I_{4}$ and using $(2.1)_{4}$, we conclude that

$$
I_{4}^{\prime}(t)=\int_{0}^{1} \rho_{2} \theta_{t t} \theta d x+\int_{0}^{1} \rho_{2} \theta_{t}^{2} d x+\int_{0}^{1} \frac{k}{2}\left(\theta_{x t} \theta_{x}+\theta_{x} \theta_{x t}\right) d x
$$




$$
\begin{aligned}
& +\int_{0}^{1} \gamma(3 W-\Psi)_{x t} \theta d x+\int_{0}^{1} \gamma(3 W-\Psi)_{x} \theta_{t} d x \\
= & \int_{0}^{1}\left[\delta \theta_{x x}-\gamma(3 W-\Psi)_{x t}+k \theta_{t x x}\right] \theta d x+\int_{0}^{1} \rho_{2} \theta_{t}^{2} d x-k \int_{0}^{1} \theta_{x x t} \theta d x \\
& +\int_{0}^{1} \gamma(3 W-\Psi)_{x t} \theta d x+\int_{0}^{1} \gamma(3 W-\Psi)_{x} \theta_{t} d x \\
= & \int_{0}^{1} \delta \theta_{x x} \theta d x+\int_{0}^{1} \rho_{2} \theta_{t}^{2} d x+\int_{0}^{1} \gamma(3 W-\Psi)_{x} \theta_{t} d x .
\end{aligned}
$$

Thanks to young's inequality, (4.2) is established.

Lemma 4.6 Let $(\Phi, \Psi, W, \theta)$ be the solution of problem (2.1)-(2.2). The functional

$$
I_{5}(t):=I_{\rho_{1}} \int_{0}^{1}(3 W-\Psi)_{t}\left(\Phi_{x}-\Psi\right) d x+\frac{D}{G} \rho_{1} \int_{0}^{1}(3 W-\Psi)_{x} \Phi_{t} d x
$$

satisfies

$$
\begin{aligned}
I_{5}^{\prime}(t) \leq & -\frac{G}{2} \int_{0}^{1}\left(\Psi-\Phi_{x}\right)^{2} d x+C \int_{0}^{t} \theta_{t x}^{2} d x+I_{\rho_{1}} \int_{0}^{1}(3 W-\Psi)_{t}^{2} d x \\
& +\varepsilon_{5} \int_{0}^{1}(3 W-\Psi)_{t}^{2} d x+C\left(\varepsilon_{5}\right) \int_{0}^{1} W_{t}^{2} d x+\left(\frac{D}{G} \rho_{1}-I_{\rho_{1}}\right) \int_{0}^{1}(3 W-\Psi)_{x t} \Phi_{t} d x .
\end{aligned}
$$

Proof. By differentiating $I_{5}$ and using $(2.1)_{2}$, we conclude that

$$
\begin{aligned}
I_{5}^{\prime}(t)= & I_{\rho_{1}} \int_{0}^{1}(3 W-\Psi)_{t t}\left(\Phi_{x}-\Psi\right) d x+I_{\rho_{1}} \int_{0}^{1}(3 W-\Psi)_{t}\left(\Phi_{x}-\Psi\right)_{t} d x \\
& +\frac{D}{G} \rho_{1} \int_{0}^{1}(3 W-\Psi)_{x t} \Phi_{t} d x+\frac{D}{G} \rho_{1} \int_{0}^{1}(3 W-\Psi)_{x} \Phi_{t t} d x \\
= & -\int_{0}^{1} G\left(\Psi-\Phi_{x}\right)^{2} d x+\int_{0}^{1} D(3 W-\Psi)_{x x}\left(\Phi_{x}-\Psi\right) d x-\int_{0}^{1} \alpha \theta_{t x}\left(\Phi_{x}-\Psi\right) d x \\
& -I_{\rho_{1}} \int_{0}^{1}(3 W-\Psi)_{t}\left(\Psi-\Phi_{x}\right)_{t} d x+\frac{D}{G} \rho_{1} \int_{0}^{1}(3 W-\Psi)_{x t} \Phi_{t} d x-D \int_{0}^{1}(3 W-\Psi)_{x}\left(\Psi-\Phi_{x}\right)_{x} d x \\
= & -\int_{0}^{1} G\left(\Psi-\Phi_{x}\right)^{2} d x+\int_{0}^{1} \alpha \theta_{t x}\left(\Psi-\Phi_{x}\right) d x+I_{\rho_{1}} \int_{0}^{1}(3 W-\Psi)_{t} \Psi_{t} d x \\
& +\left(\frac{D}{G} \rho_{1}-I_{\rho_{1}}\right) \int_{0}^{1}(3 W-\Psi)_{x t} \Phi_{t} d x .
\end{aligned}
$$

Similarly, using young's inequality, (4.3) is established. $\square$ Proof. of Theorem 2.2: To finalize the proof, we assume $\frac{G}{\rho_{1}}=\frac{D}{I_{\rho_{1}}}$ and define a Lyapunov functional $\mathcal{L}$ as follows

$$
\mathcal{L}(t):=N E(t)+I_{1}(t)+N_{2} I_{2}(t)+N_{3} I_{3}(t)+I_{4}(t)+N_{5} I_{5}(t)
$$

where $N, N_{2}, N_{3}, N_{5}$ are positive constants to be chosen properly later. Using Cauchy-Schwarz inequality and the Poincare inequality, one can easily see that all $I_{i}(t), i=1,2,3,4,5$ are bounded by an expression with the existing terms in the energy $E(t)$. This leads to the equivalence of 
$\mathcal{L}(t)$ and $E(t)$. Gathering the estimates in the previous lemmas and using $\int_{0}^{1} \theta_{t}^{2} d x \leq \int_{0}^{1} \theta_{t x}^{2} d x$, we arrive at

$$
\begin{aligned}
\mathcal{L}^{\prime}(t) \leq & -\left(4 N \gamma \beta_{2}-C-N_{5} C\left(\varepsilon_{5}\right)\right) \int_{0}^{1} W_{t}^{2} d x \\
& -\left(N \alpha G-N_{3} C\left(\varepsilon_{3}\right)-N_{5} C-N_{2} C-I_{\rho_{1}} \alpha N_{3}-N_{3} C\left(\varepsilon_{3}\right)-C\left(\varepsilon_{4}\right)-\rho_{2}\right) \int_{0}^{1} \theta_{x t}^{2} d x \\
& -\rho_{1} \int_{0}^{1} \Phi_{t}^{2} d x-\frac{2 \beta_{1}}{3} \int_{0}^{1} W^{2} d x-\frac{D}{2} \int_{0}^{1} W_{x}^{2} d x-\delta \int_{0}^{1} \theta_{x}^{2} d x \\
& -\left(\frac{G}{2} N_{5}-C-C N_{2}-\varepsilon_{3} N_{3}\right) \int_{0}^{1}\left(\Psi-\Phi_{x}\right)^{2} d x \\
& -\left(\frac{D}{2} N_{2}-C-\varepsilon_{4}\right) \int_{0}^{1}\left(3 W_{x}-\Psi_{x}\right)^{2} d x \\
& -\left(\frac{\gamma I_{\rho_{1}}}{2} N_{3}-I_{\rho_{1}} N_{2}-I_{\rho_{1}} N_{5}-N_{5} \varepsilon_{5}\right) \int_{0}^{1}\left(3 W_{t}-\Psi_{t}\right)^{2} d x
\end{aligned}
$$

At this point, we need to choose our constants carefully. First, we take $N_{2}$ large enough and $\varepsilon_{4}$ small, such that $\frac{D}{2} N_{2}-C-\varepsilon_{4}>0$. Then, we choose $N_{5}$ large enough, so that $\frac{G}{2} N_{5}-C-C N_{2}>$ 0 . Next, we pick $\varepsilon_{5}$ small and choose $N_{3}$ large enough such that $\frac{\gamma}{2} I_{\rho_{1}} N_{3}-I_{\rho_{1}} N_{2}-I_{\rho_{1}} N_{5}-N_{5} \varepsilon_{5}>$ 0 . We then select $\varepsilon_{3}$ so small that $\frac{D}{2} N_{2}-C-\varepsilon_{4}-N_{3} \varepsilon_{3}>0$ and $\frac{G}{2} N_{5}-C-C N_{2}-\varepsilon_{3} N_{3}>0$. Finally, we choose $N$ so large such that $4 N \gamma \beta_{2}-C-N_{5} C\left(\varepsilon_{5}\right)>0$ and $N \alpha G-N_{3} C\left(\varepsilon_{3}\right)-N_{5} C-$ $N_{2} C-I_{\rho_{1}} \alpha N_{3}-N_{3} C\left(\varepsilon_{3}\right)-C\left(\varepsilon_{4}\right)-\rho_{2}>0$. From the above, we deduce that for some positive constants $\gamma_{1}, \gamma_{2}$, one has $\gamma_{1} E(t) \leq \mathcal{L}(t) \leq \gamma_{2} E(t)$. Therefore, (4.5) becomes

$$
\mathcal{L}^{\prime}(t) \leq-c E(t)
$$

For $s=\frac{c}{\gamma_{2}}$, we get

$$
\mathcal{L}^{\prime}(t) \leq-s \mathcal{L}(t), \forall t \geq 0
$$

A simple integration of $(4.6)$ over $(0, t)$ leads to

$$
\mathcal{L}(t) \leq \mathcal{L}(0) e^{-s t}, \forall t \geq 0
$$

It gives the desired result Theorem 2.2 when combined with the equivalence of $\mathcal{L}(t)$ and $E(t)$.

\section{$5 \quad$ Lack of exponential stability}

In this section, by using Gearhart-Herbst-Prüss-Huang theorem [14], we give the proof of Theorem 2.3 which concludes the lack of exponential decay result. We consider that there exists a sequence of imaginary number $\lambda_{\mu}$ and functions

$$
F_{\mu}=\left(f^{1}, f^{2}, f^{3}, f^{4}, f^{5}, f^{6}, f^{7}, f^{8}\right)^{T} \in \mathcal{H},
$$


with $\left\|F_{\mu}\right\|_{\mathcal{H}} \leq 1$ such that $\left\|\left(\lambda_{\mu} I-\mathcal{A}\right)^{-1} F_{\mu}\right\|_{\mathcal{H}} \rightarrow \infty$ where

$$
\lambda_{\mu} U_{\mu}-\mathcal{A} U_{\mu}=F_{\mu}
$$

with $U_{\mu}=\left(\Phi, 3 W-\Psi, W, \theta, \Phi_{t}, 3 W_{t}-\Psi_{t}, W_{t}, \theta_{t}\right)$ not bounded. Rewrite the spectral equation (5.1) in term of its components, for $\lambda_{\mu}=\lambda$, we have

$$
\left\{\begin{array}{l}
\lambda \Phi-\Phi_{t}=f^{1}, \\
\lambda(3 W-\Psi)-(3 W-\Psi)_{t}=f^{2}, \\
\lambda W-W_{t}=f^{3} \\
\lambda \theta-\theta_{t}=f^{4} \\
\rho_{1} \lambda \Phi_{t}-G \partial_{x x} \Phi-G \partial_{x}(3 W-\Psi)+3 G \partial_{x} W=\rho_{1} f^{5}, \\
I_{\rho_{1}} \lambda(3 W-\Psi)_{t}+G \partial_{x} \Phi+G(3 W-\Psi)-3 G W-D \partial_{x x}(3 W-\Psi)+\alpha \partial_{x} \theta_{t}=I_{\rho_{1}} f^{6}, \\
I_{\rho_{1}} \lambda W_{t}-G(3 W-\Psi)+3 G W-G \partial_{x} \Phi+\frac{4}{3} \beta_{1} W+\frac{4}{3} \beta_{2} W_{t}-D \partial_{x x} W=I_{\rho_{1}} f^{7}, \\
\rho_{2} \lambda \theta_{t}-\delta \partial_{x x} \theta+\gamma \partial_{x}(3 W-\Psi)_{t}-k \partial_{x x} \theta_{t}=\rho_{2} f^{8} .
\end{array}\right.
$$

Taking $f^{1}=f^{2}=f^{3}=f^{4}=0$, we arrive at

$$
\left\{\begin{array}{l}
\rho_{1} \lambda^{2} \Phi-G \partial_{x x} \Phi-G \partial_{x}(3 W-\Psi)+3 G \partial_{x} W=\rho_{1} f^{5}, \\
I_{\rho_{1}} \lambda^{2}(3 W-\Psi)+G \partial_{x} \Phi+G(3 W-\Psi)-3 G W-D \partial_{x x}(3 W-\Psi)+\lambda \alpha \partial_{x} \theta=I_{\rho_{1}} f^{6}, \\
I_{\rho_{1}} \lambda^{2} W-G(3 W-\Psi)+3 G W-G \partial_{x} \Phi+\frac{4}{3} \beta_{1} W+\frac{4}{3} \lambda \beta_{2} W-D \partial_{x x} W=I_{\rho_{1}} f^{7}, \\
\rho_{2} \lambda^{2} \theta-\delta \partial_{x x} \theta+\lambda \gamma \partial_{x}(3 W-\Psi)-k \lambda \partial_{x x} \theta=\rho_{2} f^{8} .
\end{array}\right.
$$

Because of the boundary conditions given by (2.2), we assume that

$$
\Phi=A \cos (\mu \pi x),(3 W-\Psi)=B \sin (\mu \pi x), W=C \sin (\mu \pi x), \theta=E \cos (\mu \pi x) .
$$

Now, choosing

$$
f^{5}=b_{1} \cos (\mu \pi x), f^{6}=b_{2} \sin (\mu \pi x), f^{7}=b_{3} \sin (\mu \pi x), f^{8}=b_{4} \cos (\mu \pi x),
$$

we arrive at

$$
\left\{\begin{array}{l}
\rho_{1} \lambda^{2} A \cos (\mu \pi x)+G(\mu \pi)^{2} A \cos (\mu \pi x)-G \mu \pi B \cos (\mu \pi x)+3 G \mu \pi C \cos (\mu \pi x) \\
\quad=\rho_{1} b_{1} \cos (\mu \pi x), \\
I_{\rho_{1}} \lambda^{2} B \sin (\mu \pi x)-G \mu \pi A \sin (\mu \pi x)+G B \sin (\mu \pi x)-3 G C \sin (\mu \pi x) \\
+D(\mu \pi)^{2} B \sin (\mu \pi x)-\lambda \alpha \mu \pi E \sin (\mu \pi x) \\
=I_{\rho_{1}} b_{2} \sin (\mu \pi x), \\
I_{\rho_{1}} \lambda^{2} C \sin (\mu \pi x)-G B \sin (\mu \pi x)+3 G C \sin (\mu \pi x)+G \mu \pi A \sin (\mu \pi x) \\
+\frac{4}{3} \beta_{1} C \sin (\mu \pi x)+\frac{4}{3} \lambda \beta_{2} C \sin (\mu \pi x)+D(\mu \pi)^{2} C \sin (\mu \pi x) \\
=I_{\rho_{1}} b_{3} \sin (\mu \pi x), \\
\rho_{2} \lambda^{2} E \cos (\mu \pi x)+\delta(\mu \pi)^{2} E \cos (\mu \pi x)+\lambda \gamma \mu \pi B \cos (\mu \pi x)+k \lambda(\mu \pi)^{2} E \cos (\mu \pi x) \\
=\rho_{2} b_{4} \cos (\mu \pi x) .
\end{array}\right.
$$


Choosing $b_{1}=\frac{1}{\rho_{1}}, b_{2}=b_{3}=b_{4}=0$, we have

$$
\left\{\begin{array}{l}
\rho_{1} \lambda^{2} A+G(\mu \pi)^{2} A-G B \mu \pi+3 G \mu \pi C=1 \\
I_{\rho_{1}} \lambda^{2} B-A G \mu \pi+G B-3 G C+D(\mu \pi)^{2} B-\lambda \alpha \mu \pi E=0 \\
I_{\rho_{1}} \lambda^{2} C-G B+3 G C+G \mu \pi A+\frac{4}{3} \beta_{1} C+\frac{4}{3} \lambda \beta_{2} C+D(\mu \pi)^{2} C=0 \\
\rho_{2} \lambda^{2} E+\delta(\mu \pi)^{2} E+\lambda \gamma \mu \pi B+k \lambda(\mu \pi)^{2} E=0
\end{array}\right.
$$

Now, we take $\lambda=\lambda_{\mu}$, such that

$$
\rho_{1} \lambda^{2}+G(\mu \pi)^{2}=0 .
$$

Therefore, the above system can be written as

$$
\left\{\begin{array}{l}
-G B \mu \pi+3 G \mu \pi C=1 \\
-A G \mu \pi+\left[\left(D-\frac{I_{\rho_{1}} G}{\rho_{1}}\right)(\mu \pi)^{2}+G\right] B-3 G C-\sqrt{\frac{G}{\rho_{1}}} \alpha(\mu \pi)^{2} i E=0 \\
-G B+G \mu \pi A+\left[\left(D-\frac{I_{\rho_{1}} G}{\rho_{1}}\right)(\mu \pi)^{2}+\frac{4}{3} \beta_{1}+3 G+\frac{4}{3} \sqrt{\frac{G}{\rho_{1}}} \beta_{2} \mu \pi i\right] C=0 \\
\sqrt{\frac{G}{\rho_{1}}} \gamma(\mu \pi)^{2} i B+\left(-\frac{\rho_{2} G}{\rho_{1}}+\delta+k \sqrt{\frac{G}{\rho_{1}}} \mu \pi i\right)(\mu \pi)^{2} E=0
\end{array}\right.
$$

Add $(5.2)_{2}$ to $(5.2)_{3}$, we conclude that

$$
\left(D-\frac{I_{\rho_{1}} G}{\rho_{1}}\right)(\mu \pi)^{2} B+\left[\left(D-\frac{I_{\rho_{1}} G}{\rho_{1}}\right)(\mu \pi)^{2}+\frac{4}{3} \beta_{1}+\frac{4}{3} \sqrt{\frac{G}{\rho_{1}}} \beta_{2} \mu \pi i\right] C-\sqrt{\frac{G}{\rho_{1}}} \alpha(\mu \pi)^{2} i E=0
$$

It follows from $(5.2)_{4}$, we get

$$
E=\frac{\gamma \sqrt{\frac{G}{\rho_{1}}} i}{\frac{\rho_{2} G}{\rho_{1}}-\delta-k \sqrt{\frac{G}{\rho_{1}}}(\mu \pi) i} B
$$

Combining (5.4) and (5.3) yields

$$
C=\frac{N}{M} B
$$

where

$$
\begin{gathered}
N=\frac{\alpha \pi \gamma G}{\mu \rho_{1}}\left(\frac{\rho_{2} G}{\rho_{1}}-\delta-k \sqrt{\frac{G}{\rho_{1}}}(\mu \pi) i\right)^{-1}-\left(D-\frac{I_{\rho_{1}} G}{\rho_{1}}\right)(\mu \pi)^{2}, \\
M=\left(D-\frac{I_{\rho_{1}} G}{\rho_{1}}\right)(\mu \pi)^{2}+\frac{4}{3} \beta_{1}+\frac{4}{3} \sqrt{\frac{G}{\rho_{1}}} \beta_{2}(\mu \pi) i .
\end{gathered}
$$

Subtracting $C$ into $(5.2)_{3}$ and $(5.2)_{1}$, we get

$$
A=\frac{G-(M+3 G) \frac{N}{M}}{G(\mu \pi)} B, \quad B=\frac{M}{(3 N-M) G(\mu \pi)} .
$$


When $\mu \rightarrow \infty$, we get $B(\mu \pi) \rightarrow-\frac{1}{4 G}$. Then, substituting $B$ into $A, C, E$, we obtain

$$
A \rightarrow-\frac{1}{4}, C \rightarrow o\left(\frac{1}{\mu}\right), E \rightarrow o\left(\frac{1}{\mu}\right) .
$$

Thus

$$
\begin{aligned}
\left\|U_{\mu}\right\|_{\mathcal{H}}^{2} \geq & G \int_{0}^{1}\left(\Psi-\Phi_{x}\right)^{2} d x=G \int_{0}^{1}(-B+3 C+\mu \pi A)^{2} \sin ^{2}(\mu \pi x) d x \\
& =G A^{2}(\mu \pi)^{2} \int_{0}^{1} \sin ^{2}(\mu \pi x) d x=\frac{1}{2} G A^{2}(\mu \pi)^{2}-\frac{1}{4} G A^{2}(\mu \pi) \sin (2 \mu \pi x) \begin{array}{l}
x=1 \\
x=0
\end{array} \\
& \rightarrow \infty \text { as } \mu \rightarrow \infty .
\end{aligned}
$$

Therefore, this completes the proof.

\section{Polynomial decay for $\frac{G}{\rho_{1}} \neq \frac{D}{I_{\rho_{1}}}$}

In this section, we prove the polynomial decay result for the case $\frac{G}{\rho_{1}} \neq \frac{D}{I_{\rho_{1}}}$. Similar as Lemma 4.1 , a simple calculation about the second-order energy implies

$$
\frac{d}{d t} E_{2}(t)=-4 \gamma \int_{0}^{1} \beta_{2} W_{t t}^{2} d x-\alpha \int_{0}^{1} k \theta_{x t t}^{2} d x \leq 0 .
$$

Now, we prove Theorem 2.4. Let $\frac{G}{\rho_{1}}-\frac{D}{I_{\rho_{1}}} \neq 0$, the last term in (4.4) can be handled as follows. Using $(2.1)_{4}$ and Young's inequality, we obtain

$$
\begin{aligned}
\int_{0}^{1}(3 W-\Psi)_{x t} \Phi_{t} d x= & \frac{1}{\gamma} \int_{0}^{1}\left(k \theta_{x x t}+\delta \theta_{x x}-\rho_{2} \theta_{t t}\right) \Phi_{t} d x \\
= & -\frac{\rho_{2}}{\gamma} \int_{0}^{1} \theta_{t t} \Phi_{t} d x+\frac{\delta}{\gamma} \int_{0}^{1} \theta_{x x} \Phi_{t} d x+\frac{k}{\gamma} \int_{0}^{1} \theta_{x x t} \Phi_{t} d x \\
= & -\frac{\rho_{2}}{\gamma} \int_{0}^{1} \theta_{t t} \Phi_{t} d x+\frac{\delta}{\gamma} \int_{0}^{1} \theta_{x t} \Phi_{x} d x+\frac{k}{\gamma} \int_{0}^{1} \theta_{x t t} \Phi_{x} d x \\
& -\frac{d}{d t} \int_{0}^{1}\left(\frac{\delta}{\gamma} \theta_{x} \Phi_{x}+\frac{k}{\gamma} \theta_{x t} \Phi_{x}\right) d x
\end{aligned}
$$

Multiplying by $\frac{D}{G} \rho_{1}-I_{\rho_{1}}$, we get

$$
\begin{aligned}
\left(\frac{D}{G} \rho_{1}-I_{\rho}\right) \int_{0}^{1}(3 W-\Psi)_{x t} \Phi_{t} d x \leq & -\frac{d}{d t}\left(\frac{D}{G} \rho_{1}-I_{\rho_{1}}\right) \int_{0}^{1}\left(\frac{\delta}{\gamma} \theta_{x} \Phi_{x}+\frac{k}{\gamma} \theta_{x t} \Phi_{x}\right) d x \\
& +\varepsilon_{6} \int_{0}^{1}\left(\Phi_{t}^{2}+\Phi_{x}^{2}\right) d x+C\left(\varepsilon_{6}\right) \int_{0}^{1}\left(\theta_{x t}^{2}+\theta_{x t t}^{2}\right) d x
\end{aligned}
$$

where we have used that

$$
\begin{aligned}
\Phi_{x}^{2}=\left(\Phi_{x}-\Psi+\Psi\right)^{2} & \leq 2\left(\Phi_{x}-\Psi\right)^{2}+2(\Psi-3 W+3 W)^{2} \\
& \leq 2\left(\Phi_{x}-\Psi\right)^{2}+4(\Psi-3 W)^{2}+36 W^{2} .
\end{aligned}
$$


We then define

$$
L(t)=\bar{L}(t)+\left(\frac{D}{G} \rho_{1}-I_{\rho_{1}}\right) \int_{0}^{1}\left(\frac{\delta}{\gamma} \theta_{x} \Phi_{x}+\frac{k}{\gamma} \theta_{x t} \Phi_{x}\right) d x
$$

where

$$
\bar{L}(t)=N\left(E(t)+E_{2}(t)\right)+I_{1}(t)+N_{2} I_{2}(t)+N_{3} I_{3}(t)+I_{4}(t)+N_{5} I_{5}(t) .
$$

From (4.5) and (2.4), we get

$$
\begin{aligned}
L^{\prime}(t) \leq & -c E(t)+\varepsilon_{6} \int_{0}^{1}\left[\Phi_{t}^{2}+2\left(\Phi_{x}-\Psi\right)^{2}+4(\Psi-3 W)^{2}+36 W^{2}\right] d x \\
& -\left(N-C\left(\varepsilon_{6}\right)\right) \int_{0}^{1} \theta_{x t t}^{2} d x-\left(N-C\left(\varepsilon_{6}\right)\right) \int_{0}^{1} \theta_{x t}^{2} d x .
\end{aligned}
$$

Then we choose $\varepsilon_{6}$ small enough and take $N$ large enough so that $L$ is positive and

$$
N \alpha G-N_{3} C\left(\varepsilon_{3}\right)-N_{5} C-N_{2} C-I_{\rho} \alpha N_{3}-N_{3} C\left(\varepsilon_{3}\right)-C\left(\varepsilon_{4}\right)-\rho_{2}-C\left(\varepsilon_{6}\right)>0 .
$$

Depending on above constants, we deduce that

$$
L^{\prime}(t) \leq-\frac{c}{2} E(t)
$$

Integrating over $(0, t)$, we have

$$
t E(t) \leq \int_{0}^{t} E(s) d s \leq \frac{2}{c}\left(L^{\prime}(0)-L^{\prime}(t)\right) \leq \frac{2 L^{\prime}(0)}{c} .
$$

Consequently,

$$
E(t) \leq \frac{2 L(0)}{c t} \leq \frac{C_{1}\left(E(0)+E_{2}(0)\right)}{t} \quad \forall t>0 .
$$

This completes the proof.

\section{Acknowledgements}

This work was supported by the National Natural Science Foundation of China [grant number 11771216], the Natural Science Foundation of Jiangsu Province [grant number BK20151523], the Six Talent Peaks Project in Jiangsu Province [grant number 2015-XCL-020] and the Qing Lan Project of Jiangsu Province.

\section{References}

[1] T. A. Apalara, Uniform stability of a laminated beam with structural damping and second sound, Z. Angew. Math. Phys. 68 (2017), no. 2, 68:41.

[2] X.-G. Cao, D.-Y. Liu and G.-Q. Xu, Easy test for stability of laminated beams with structural damping and boundary feedback controls, J. Dyn. Control Syst. 13 (2007), no. 3, $313-336$. 
[3] M. M. Cavalcanti et al., Uniform decay rates for the energy of Timoshenko system with the arbitrary speeds of propagation and localized nonlinear damping, Z. Angew. Math. Phys. 65 (2014), no. 6, 1189-1206.

[4] M. M. Chen, W. J. Liu and W. C. Zhou, Existence and general stabilization of the Timoshenko system of thermo-viscoelasticity of type III with frictional damping and delay terms, Adv. Nonlinear Anal., in press. doi:10.1515/anona-2016-0085

[5] S. Drabla, S. A. Messaoudi and F. Boulanouar, A general decay result for a multidimensional weakly damped thermoelastic system with second sound, Discrete Contin. Dyn. Syst. Ser. B 22 (2017), no. 4, 1329-1339.

[6] A. Fareh and S. A. Messaoudi, Stabilization of a type III thermoelastic Timoshenko system in the presence of a time-distributed delay, Math. Nachr. 290 (2017), no. 7, 1017-1032.

[7] J. A. Goldstein, Semigroups of linear operators and applications, Oxford Mathematical Monographs, The Clarendon Press, Oxford University Press, New York, 1985.

[8] A. E. Green and P. M. Naghdi, A re-examination of the basic postulates of thermomechanics, Proc. Roy. Soc. London Ser. A 432 (1991), no. 1885, 171-194.

[9] S. W. Hansen. A model for a two-layered plate with interfacial slip. Control and estimation of distributed parameter systems: nonlinear phenomena (Vorau, 1993), 143-170, Internat. Ser. Numer. Math. vol. 118, Birkhauser, Basel, 1994.

[10] S. W. Hansen and R. Spies, Structural damping in laminated beams due to interfacial slip, J. Sound Vibration. 204 (1997), no. 2, 183-202.

[11] J. Hao and P. Wang, Asymptotical stability for memory-type porous thermoelastic system of type III with constant time delay, Math. Methods Appl. Sci. 39 (2016), no. 13, 3855-3865.

[12] J. Hao and P. Wang, Exponential decay of solution to the viscoelastic porous-thermoelastic system of type III with boundary time-varying delay, Math. Methods Appl. Sci. 39 (2016), no. $13,3659-3668$.

[13] M. Kafini et al., Well-posedness and stability results in a Timoshenko-type system of thermoelasticity of type III with delay, Z. Angew. Math. Phys. 66 (2015), no. 4, 1499-1517.

[14] A. A. Keddi, T. A. Apalaras and S. A. Messaoudi, Exponential and polynomial decay in a thermoelastic-Bresse system with second sound, Appl. Math. Optim., in press. DOI:10.1007/s00245-016-9376-y

[15] G. Li, X. Y. Kong and W. J. Liu, General decay for a laminated beam with structural damping and memory: the case of non-equal wave speeds, J. Integral Equations Appl., in press. 
[16] W. J. Liu, K. W. Chen and J. Yu, Existence and general decay for the full von Kármán beam with a thermo-viscoelastic damping, frictional dampings and a delay term, IMA J. Math. Control Inform. 34 (2017), no. 2, 521-542.

[17] W. J. Liu, K. W. Chen and J. Yu, Asymptotic stability for a non-autonomous full von Kármán beam with thermo-viscoelastic damping, Appl. Anal., in press. doi: 10.1080/00036811.2016.1268688

[18] W. J. Liu and W. F. Zhao, Stabilization of a thermoelastic laminated beam with past history, Appl. Math. Optim., in press. doi: 10.1007/s00245-017-9460-y

[19] A. Lo and N. Tatar, Uniform Stability of a Laminated Beam with Structural Memory, Qual. Theory Dyn. Syst. 15 (2016), no. 2, 517-540.

[20] A. Lo and N. Tatar, Stabilization of laminated beams with interfacial slip, Electron. J. Differential Equations 2015 (2015), No. 129, 14 pp.

[21] S. A. Messaoudi and T. A. Apalara, General stability result in a memory-type porous thermoelasticity system of type III, Arab J. Math. Sci. 20 (2014), no. 2, 213-232.

[22] S. A. Messaoudi and A. Fareh, Energy decay in a Timoshenko-type system of thermoelasticity of type III with different wave-propagation speeds, Arab. J. Math. (Springer) 2 (2013), no. 2, 199-207.

[23] S. A. Messaoudi and B. Said-Houari, Energy decay in a Timoshenko-type system of thermoelasticity of type III, J. Math. Anal. Appl. 348 (2008), no. 1, 298-307.

[24] M. I. Mustafa, On the decay rates for thermoviscoelastic systems of type III, Appl. Math. Comput. 239 (2014), 29-37.

[25] A. Pazy, Semigroups of linear operators and applications to partial differential equations. Springer, New York, 1983.

[26] Y. Qin, X.-G. Yang and Z. Ma, Global existence of solutions for the thermoelastic Bresse system, Commun. Pure Appl. Anal. 13 (2014), no. 4, 1395-1406.

[27] B. Said-Houari and T. Hamadouche, The Cauchy problem of the Bresse system in thermoelasticity of type III, Appl. Anal. 95 (2016), no. 11, 2323-2338.

[28] F. Tahamtani, On energy decay of an $n$-dimensional thermoelasticity system with a nonlinear weak damping, Iran. J. Sci. Technol. Trans. A Sci. 32 (2008), no. 1, 45-51, 83.

[29] N.-E. Tatar, Stabilization of a laminated beam with interfacial slip by boundary controls, Bound. Value Probl. 2015, 2015:169, 11 pp. 
[30] Z. Tian and G.-Q. Xu. Exponential stability analysis of Timoshenko beam system with boundary delays. Appl. Anal. (2016), 1-29.

[31] J.-M. Wang, G.-Q. Xu and S.-P. Yung, Exponential stabilization of laminated beams with structural damping and boundary feedback controls, SIAM J. Control Optim. 44 (2005), no. $5,1575-1597$. 